\title{
Les fuites \\ des galeries en charge en terrain sec Rôle du revêtement, des injections, du terrain
}

PAR

\author{
M. Bouvard \\ Directeur de l'Ecole Nationale Superieure d'Hydraulique \\ de Grenoble
}

\section{1 - L'équilibre hydraulique des galeries en charge}

\section{A) LES CONCEPTIONS ACTUELLES}

Après une phase où on a considéré que l'équilibre des galeries en charge (étanchéité, tenue mécanique) correspondait à l'application de la pression à la surface de la galerie, il est désormais couramment admis que ces phénomènes sont régis essentiellement par des aspects hydrogéologiques complexes, où les écoulements globaux dans le massif rocheux jouent un rôle fondamental. Quant aux efforts mécaniques résultant de la pression intérieure, ils sont étroitement conditionnés par les pertes de charge dans les écoulements à travers les différents milieux : revêtement, zone injectée, rocher, etc., qui limitent la galerie en pression. Notamment, s'il existait avant perforation de la galeric une nappe dans le rocher à une pression supérieure à celle de la galerie, on peut considérer qu'il ne se posera pratiquement aucun problème quant aux fuites de la galerie.

Au point de vue expérimental, les essais de la galerie en charge Dixence-Bagnes (cf. Bulletin technique de la Suisse romande, janvier 1958), de la galerie de Roselend (cf. La Houille Blanche, $\mathrm{n}^{\circ}$ spécial $\mathrm{B}$ /1962) apportaient d'importantes contributions à l'appui de cette conception.

Mais, dans certains cas, il n'est pas possible de placer les galeries en charge dans une zone où la pression de l'eau dans le rocher est supérieure à la pression qui règnera à l'intérieur de la galerie. A la limite, on est quelquefois obligé d'implanter les galeries en charge dans une zone «sèche ». Le cas peut évidemment se produire, notamment si la galerie est localisée à une hauteur trop grande par rapport aux vallées voisines, ou très près de la surface (le niveau de la nappe n'a pas encore atteint le niveau de la galerie), ou si le terrain présente des anomalies de perméabilité. C'est le cas notamment des terrains calcaires où on peut avoir simultanément:

- une perméabilité locale relativement faible;

- une perméabilité d'ensemble considérable, notamment si le terrain est « karstifié »: les canaux karstiques représentent alors un réseau de drainage qui empêche la nappe de se former.

C'est un problème de ce type que le soussigné a été amené à examiner à propos de l'étude d'une galerie en charge (de 60 à $80 \mathrm{~m}$ de pression) implantée dans des terrains calcaires partiellement karstifiés, ou dans des zones sans nappe extérieure.

Le phénomène doit alors être analysé sur d'autres bases que celles correspondant aux galeries construites dans des terrains baignés par une nappe.

I.a présente étude se propose d'examiner les conditions de fonctionnement ou d'étanchéité de la galerie:

\section{- D'abord du point de vue hydraulique:}

L'hydraulique des écoulements poreux dans le rocher continue à jouer le même rôle fondamental que s'il existait une nappe (mais bien sûr sous une autre forme). Un calcul nous permettra de proposer un schéma théoriquement exact de ces phénomènes conduisant à calculer les fuites d'une galerie en terrain «sec», de perméabilité donnée. Ce calcul sera étendu sans difficultés au cas d'un terrain de perméabilité variable, constante dans des cylindres concentriques à l'axe de la galerie. 
-- Ensuite au point de vue mécanique:

La charge à l'intérieur de la galerie se transforme alors en pertes de charge des écoulements poreux dans les divers milieux ainsi constitués. Cette perte de charge «répartie» entraîne l'apparition de contraintes mécaniques. Les milieux se déforment, se fissurent, ce qui modifie leur perméabilité, et, à nouveau, redistribuent les pertes de charge, puis les efforts.

Après examen qualitatif et quantitatif de ces problèmes, nous proposerons une série de conclusions concernant les solutions à envisager pour résoudre les difficultés qui en résulteraient.

\section{B) CONTRIBUTION ANTERIEURE}

Dans cette étude, nous serons amenés à nous référer à des publications antérieures dont nous rappellerons brièvement ci-dessous les références :

1) Pressions interstitielles dans les galeries en charge, de G. Sauvage de Saint-Marc, M. Bouvard, Ma Min YuAn (La Houille Blanche, mars-avril 1960).

On analysait les effets de la «diffusion » des pressions intérieures, sous l'action de l'écoulement poreux provoqué par la pression intérieure dans l'enveloppe qui contient l'eau en pression (d'épaisseur finie s'il s'agit d'une canalisation en béton, ou pratiquement infinie s'il s'agit d'une galerie) surtout sous l'aspect des contraintes, tandis que, dans la seconde partie, on mettait en évidence le rôle fondamental de l'écoulement dans le terrain, sous son aspect au moins qualitatif.

2) Le puits en charge de l'aménagement Capivari-Cachoeira (La Houille Blanche, $\mathrm{n}^{\circ}$ 7/1969);

3) Les ouvrages en charge de l'aménagement CapivariCachoeira (La Houille Blanche, $\mathrm{n}^{\circ} 4 / 1971$ ). de M. Bouvard et N. Pinto,

où les auteurs présentaient plusieurs résultats et analyses théoriques.

\section{Puits en CHARgE}

Il comprend une partie non blindée, suivie d'une partie munie d'un blindage en acier.

Partie non hlindée. - La pression statique atteint $450 \mathrm{~m}$ (pression dynamique: $500 \mathrm{~m}$ ). Encore aujourd'hui, cet ouvrage, de $3,00 \mathrm{~m}$ de diamètre, constitue le record du monde des ouvrages en pression sans blindage. Dans cette publication, les auteurs présentent les études, essais préliminaires, les principes adoptés dans l'établissement du projet, Ies mesures effectuées à la mise en service.

Partie blindée. - On avait notamment prévu, sur ce puits blindé (charge statique: $750 \mathrm{~m}$ ), une galerie de drainage, visitable, équipée de drains secondaires, qui a permis de procéder à des mesures fort intéressantes pour le cas des ouvrages en pression, blindés ou non.

\section{Galerie en charge}

Autre solution originale semble-t-il: le revêtement de cette galerie a été, non pas injecté comme le voulaient alors les règles de l'art, mais rendu systématiquement perméable à l'aide de très nombreux forages percés à travers le béton. Un calcul, utilisant la théoric des écoulements poreux, permettait de déterminer la part prise par le revêtement dans une galerie en charge soumise à des fluctuations de pression lorsque, après une longue période d'exploitation, les pressions dans le terrain et dans la galerie se sont équilibrées.

En particulier, cette étude propose une formule qui permet de déterminer les fuites initiales en régime transitoire dans une galerie lorsque la pression augmente de $\Delta H$, la nappe dans le terrain étant équilibrée avec la pression intérieure $\left({ }^{1}\right)$ :

$$
Q=\frac{2 K \Delta H}{\log b / a\left[1+\sqrt{\left.1-\left(a^{2} / b^{2}\right)\right]}\right.}
$$

qui permettait de préciser ensuite la part de pression prise par le revêtement, compte tenu de la perméabilité relative du terrain et du revêtement (fig. 1).

En tout cas, près de cinq ans d'exploitation de ces deux ouvrages, sans incident, semblent avoir montré l'intérêt des dispositions adoptées et des conceptions utilisées pour les établir.

Comme nous l'avons dit ci-dessus, nous allons maintenant appliquer ces idées au cas d'une galerie en pression dans un «terrain sec».

\section{2 - Calcul des pertes des galeries en charge dans un terrain sec}

Nous adopterons bien entendu les hypothèses classiques quant aux caractéristiques du terrain. La perméabilité du rocher, considérée comme homogène, isotrope, sera délinie par le coefficient classique $K$.

L'écoulement sera supposé plan.

Ces hypothèses ne sont que peu restrictives par rapport à la réalité (2). La difficulté consisterait plus à choisir la méthode de mesure qui permettrait de définir la valeur à adopter. Aussi, comme dans le cadre des études classiques, nous considérerons que cette valeur est définie avec une précision qui permet seulement d'approcher la puissance de 10.

La galerie elle-même sera supposée circulaire. D'une part, e'est le type de section le plus souvent adopté dans ce type d'ouvrage et, d'autre part, des considérations qualitatives montrent que cette hypothèse n'est pratiquement pas restrictive si on appliquait les résultats à une galerie de forme pas trop différente, en «fer à cheval » par exemple.

(1) On peut noter que les conditions d'établissement de cette formule correspondent au cas thćorique d'une nappe horizontale drainée ì une profondeur $b$ par une canalisation de diamètre $a$. Il suffit d'introduire, au lieu de $\Delta \mathrm{H}$, la profondeur $b$.

(2) Sauf si - en ce qui concerne le caractère plan de l'écoulement - les fuites contribuaient à l'apparition d'une nappe dont la débitance serait contrôlée à l'aval, par les conditions hydrogéologiques globales du massif. Nous reviendrons sur ce point en conclusion. 


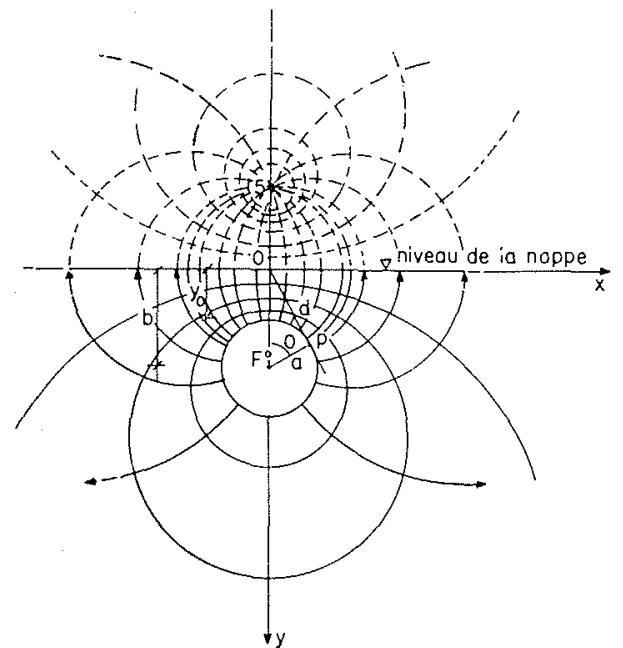

1/

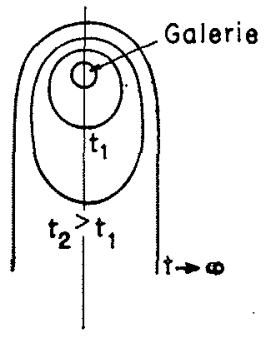

2!

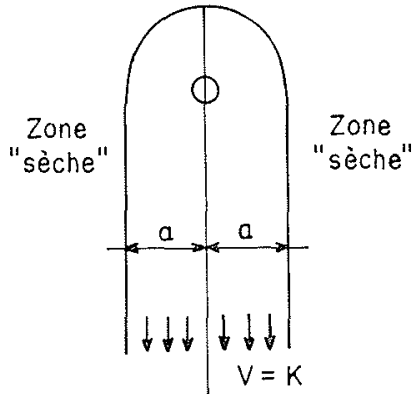

$3 /$

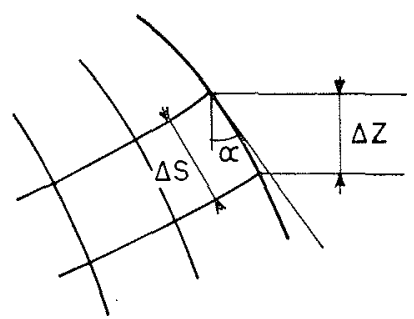

4/

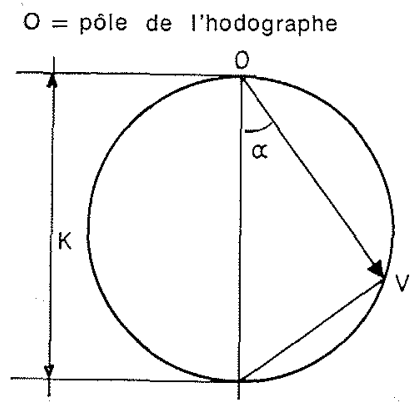

$\mathrm{OV}=\mathrm{K} \cos \alpha$

\section{A) TRACÉ DU RÉSEAU D'ÉCOULEMENT} SUIVI PAR LES FUITES

\section{$1^{\circ}$ Considérations qualitatives}

Dans la première des études antérieures citée ci-dessus (1960) nous avions indiqué un schéma qualitatif, conduisant à une conception de l'écoulement des fuites dans le rocher. $\mathrm{Si}$ on part de la galerie remplie et mise brusquement en pression, le réseau d'écoulement passe par les phases successives définies schématiquement ci-dessous.

D'abord, les fuites contribuent à remplir une auréole de terrain de dimension croissante avec le temps $t$.

Puis, si l'écoulement se poursuit, l'auréole prend une forme parabolique: le débit de fuite s'écoule verticalement vers le bas (fig. 2)

On tend alors vers un régime permanent, dans la mesure évidemment où, soit la nappe, soit un niveau imperméable, serait assez loin en dessous de la galerie pour ne pas influencer l'écoulement.

C'est plus particulièrement la «ligne de saturation» de l'écoulement que nous allons considérer, c'est-à-dire la ligne (puisqu'il s'agit d'un écoulement plan, cette ligne représente une surface cylindrique) qui sépare la zone sèche de la zone intéressée par l'écoulement des fuites dans le rocher.

Quelques considérations sont à la base de la défnition de ce réseau (fig. 3).

a) Dans la partie où l'écoulement poreux est sensiblement vertical, la vitesse devient égale à la perméabilité du terrain $K$. En effet, l'écoulement est tel que la perte de potentiel de l'eau sert juste à compenser la perte de charge. Si on appelle a la largeur de la bande limitée par les asymptotes verticales à la «ligne de saturation » de l'écoulement, le débit de fuite sera tel que :

$$
Q=K a
$$

$Q=$ débit de fuite au mètre linéaire.

b) Dans la partie courante de la ligne de saturation, il va être possible de déterminer une propriété remarquable de l'écoulement qui permettra de remonter jusqu'à sa déflnition théorique.

Un tel écoulement peut bien entendu être représenté par le tracé - classique - des lignes de courant et des équipotentielles. Considérons alors la projection d'un des carrés que forment sur la ligne de saturation les lignes de courant et les équipotentielles (fig. 4).

La perte de charge sur la ligne qui limite ce carré est bien entendu égale à :

$$
(V / K) \Delta S
$$

Tandis que cette même perte de charge est égale à la perte de potentiel de l'eau sur le trajet $\Delta S$. Soit $\Delta Z$ la projection de $\Delta S$ sur la verticale. On aura donc:

$$
(V / K) \Delta S=\Delta Z
$$

Soit :

$$
V=K(\Delta \mathrm{Z} / \Delta S)
$$


Mais $(\Delta Z / \Delta S)=\cos \alpha$. Par conséquent :

$$
V=K \cos \alpha
$$

Si on considère l'hodographe de l'écoulement, à la surface de saturation, l'extrémité du vecteur vitesse décrira donc un cercle de diamètre $K$ (fig. 5).

$\mathrm{La}$ vitesse sur la ligne de saturation est nulle au sommet, où la tangente est horizontale $(\cos \alpha$ est nul) et égale à $K$ lorsque la direction de l'écoulement est devenue sensiblement verticale.

La détermination du réseau qui représentera l'écoulement se ramène donc à trouver l'expression analytique d'un réseau dont l'hodographe comprendrait un cercle d'un certain diamètre, que l'on prendrait égal à $K$. Les lignes de courant à l'intérieur ou à l'extérieur de la ligne de saturation correspondront alors, sur l'hodographe, à des courbes intérieures ou extérieures à ce même cercle.

c) Au voisinage de la galerie:

A priori - et nous verrons que cette idée se confirme dans la suite de l'étude - la hauteur $b$, atteinte par l'écoulement au-dessus de la galerie de rayon $R_{0}$, est inférieure à la pression à l'intérieur de la galerie, la différence représentant la perte de charge sur la verticale qui passe par l'axe de la galerie. Mais cette hauteur doit être a priori de l'ordre de grandeur de la charge intérieure. Or le calcul est intéressant seulement dans le cas où la charge est élevée, c'est-à-dire où $H / R_{0}$ est supérieure à des valeurs de $30 \mathrm{ou}$ 40 , ou même plus.

Dans ces conditions, on peut considérer que l'écoulement dans le rocher, au voisinage de la galerie, correspond à celui d'une source placée sur son axe. Les équipotentielles au voisinage du point-source sont alors représentées par des cercles qui se déforment à mesure qu'on s'éloigne de la source. Ils ne peuvent théoriquement se confondre avec la circonférence qui représente le profil de la galerie. Mais c'est néanmoins une de ces équipotentielles que nous prendrons comme périmètre de la galerie, l'écart étant infime et sans signification technique.

Nous avions pensé un moment que le réseau défini par une source dans un écoulement parallèle infini correspondait au réseau cherché. S'il lui correspond qualitativement, on constate facilement par le calcul que l'hodographe n'est pas un cercle. Mais il apporte une aide précieuse pour trouver le bon écoulement (fig. 6).

Or, le réseau d'écoulement plan d'une source de débit $2 q$ et d'un puits de débit $q$, que nous avions eu à traiter autrefois, met en évidence une ligne de courant singulière représentée par un cercle: il sépare la portion du débit $q$ de la source qui va vers le puits, de l'autre, $q$, qui va à l'infini. Effectivement, on constate que ce réseau est l'hodographe - quand le pôle est diamétralement opposé à la source - de l'écoulement d'une source de débit $Q$, placé entre deux plaques parallèles distantes de $2 a$ à laquelle se superpose un écoulement parallèle, tel que la vitesse à lamont soit égale à $K / 2$, et la vitesse à l'aval égale à $K$. Cet écoulement présente une ligne de séparation entre le fluide originaire de la source et celui qui vient de l'amont. Les vitesses sur la ligne de séparation correspondent, sur l'hodographe, au cercle qui constitue la ligne singulière du réseau (fig. 7). Elles respectent donc la priorité fondamentale mise en évidence ci-dessus, soit:

$$
V=K \cos \alpha
$$

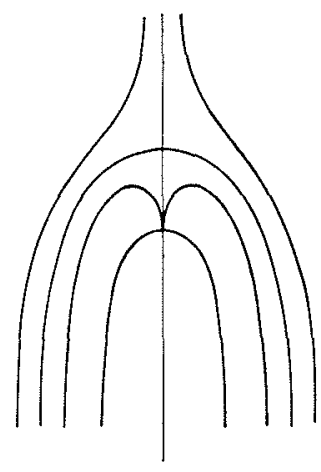

6)
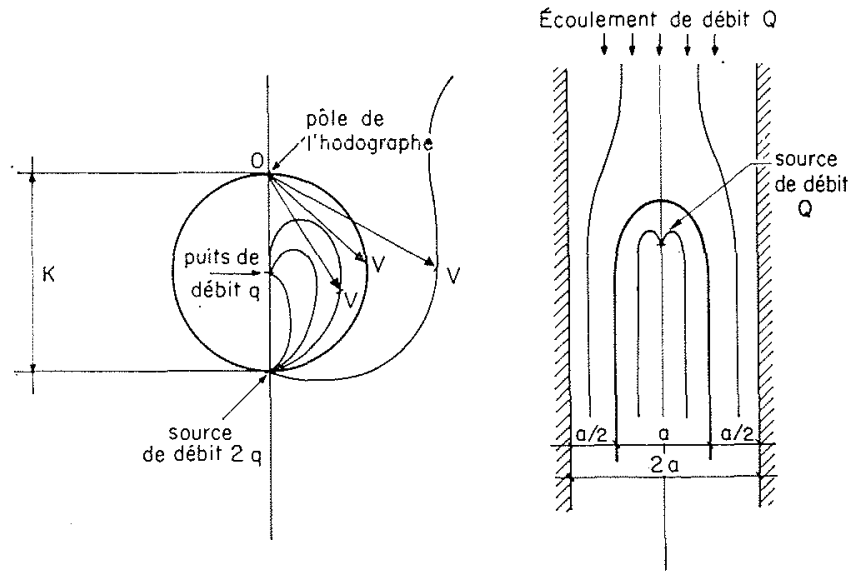

$7 /$

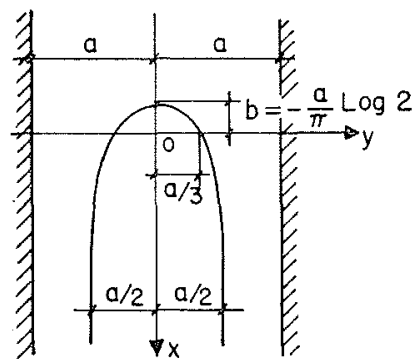

8/

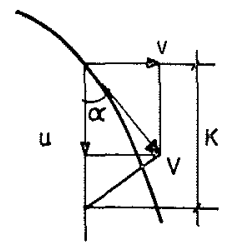

9/

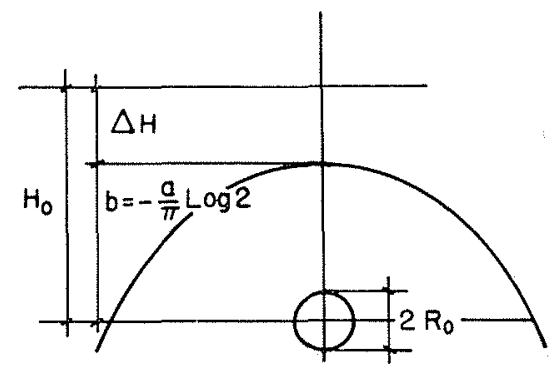

$10 /$ 
$2^{\circ}$ Expression analytique de l'écoulement ci-dessus (3)

a) Fonction complexe

Elle correspond à l'expression algébrique :

$$
f(z)=(Q / 2 \pi) \log \operatorname{sh}(\pi z / 2 a)+(3 Q z / 4 a)
$$

Elle résulte de l'addition du réseau représentant une source entre deux plaques, ( $1^{\mathrm{er}}$ terme du membre de droite) et de l'écoulement parallèle ( $2^{\mathrm{e}}$ terme), combinés pour avoir les vitesses à l'infini, à l'amont et à l'aval de la source, égales respectivement à $K / 2$ et à $K$.

On détermine la ligne de courant singulière recherchée par le fait que, si $x \rightarrow+\infty, Y \rightarrow a / 2$ (du fait des débits respectifs de la source et de l'écoulement parallèle, la ligne de séparation est asymptote à la droite $y=a / 2$ ) ce qui conduit à donner à la ligne de séparation l'équation suivante :

$$
\operatorname{tg}^{2} \eta=\frac{3 \operatorname{sh} \xi+\operatorname{ch} \xi}{3 \operatorname{ch} \xi+\operatorname{sh} \xi}
$$

avec $\eta=\pi y / 2 a$ et $\xi=\pi x / 2 a$

ou encore:

$$
\text { th } \xi=\frac{3 \sin ^{2} \eta-\cos ^{2} \eta}{3 \cos ^{2} \eta-\sin ^{2} \eta}
$$

On en déduit immédiatement les points caractéristiques suivants (fig. 8):

$$
\begin{array}{ll}
x=0 & y=a / 3 \\
y=0 & x=b=-(a / \pi) \log 2
\end{array}
$$

\section{b) VITESSES}

Les vitesses s'obtiennent en écrivant, suivant le résultat classique :

$$
d f(z) / d z=u-i v
$$

qui conduit à :

$$
\begin{aligned}
& u=\frac{Q}{8 a} \frac{\operatorname{sh} 2 \xi}{\operatorname{ch}^{2} \xi-\cos ^{2} \eta}+\frac{3 Q}{4 a} \\
& v=\frac{Q}{8 a} \frac{\sin 2 \eta}{\operatorname{ch}^{2} \xi-\cos ^{2} \eta}
\end{aligned}
$$

avec, en outre, $Q=K a$.

Nous avons vérifié que l'écoulement ainsi défini cadrait bien avec les propriétés du réseau recherché de la manière suivante :

- On calcule $v^{2}$ à partir de la dérivée $d y / d x$ telle que :

$$
\begin{aligned}
\operatorname{tg}^{2} \alpha=\left(\frac{d y}{d x}\right)^{2} & =\frac{1}{(\operatorname{sh}+\operatorname{ch})^{2}(3 \operatorname{ch}+\operatorname{sh})(3 \operatorname{sh}+\mathrm{ch})} \\
\operatorname{sh} & =\operatorname{sh} \xi \quad \operatorname{ch}=\operatorname{ch} \xi
\end{aligned}
$$

en écrivant :

$$
v^{2}=K^{2}\left[\operatorname{tg}^{2} \alpha /\left(1+\operatorname{tg}^{2} \alpha\right)^{2}\right]
$$

(3) Les caractéristiques de ce réseau avaient fait l'objet d'une étude théorique et expérimentale - non publiée - dans : «Injection ponctuelle d'un fluide pesant en milieux poreux bidimensionnels $*$ S. Inthavong. Thèse de Doctorat d'Université, Grenoble. expressions déduites des propriétés que doivent respecter $u, v$ sur la ligne de saturation (fig. 9).

On aboutit à :

$$
v^{2}=K^{2} \frac{(\mathrm{sh}+\mathrm{ch})^{2}(3 \mathrm{sh}+\mathrm{ch})(3 \mathrm{ch}+\mathrm{sh})}{\left[(\mathrm{sh}+\mathrm{ch})^{2}(3 \mathrm{sh}+\mathrm{ch})(3 \mathrm{ch}+\mathrm{sh})+1\right]^{2}}
$$

- On calcule $v^{2}$ directement, à partir de la formule donnée ci-dessus, où on a introduit $y(x)$ correspondant à la ligne de saturation, et on aboutit à :

$$
v^{2}=1 / 16 \frac{K^{2}(3 \mathrm{sh}+\mathrm{ch})(3 \mathrm{ch}+\mathrm{sh})}{\left(4 \mathrm{ch}^{3}+4 \mathrm{sh} \mathrm{ch}^{2}-3 \mathrm{sh}-\mathrm{sh}\right)^{2}}
$$

- Un calcul laborieux mais sans difficulté montre que ces deux expressions sont équivalentes. On a donc $V=K \cos \alpha$ sur la ligne de séparation qui représente bien, par conséquent, l'écoulement recherché.

\section{B) CALCUL DES PERTES D'UNE GALERIE DANS UN TERRAIN SEC}

L'expression analytique des vitesses indiquée ci-dessus va nous permettre de calculer les pertes d'une galerie creusée dans un terrain sec.

Localement, près de la source, les équipotentielles sont représentées par des cercles peu déformés, et nous allons, comme nous l'avons indiqué ci-dessus, assimiler l'un d'eux au cercle de rayon $R_{0}$ qui représente la section de la galerie.

\section{1" Calcul de la perte de charge de l'écoulement poreux : Cas d'un terrain homogène : galerie sans revêtement}

Pour le moment, nous connaissons $H_{0}$, charge de la galerie, $R_{0}$, K. Le paramètre a est encore inconnu. Sa détermination permettra de calculer les fuites qui, conformément à la définition du réseau, sont égales à $K a$ (fig. 10).

Nous allons écrire :

$$
H_{0}-b=\Delta H=\int_{R_{11}}^{b}(u / K) d x
$$

L'intégrale se calcule sans difficulté. Du fait que $y=0$ sur l'axe, l'expression de la vitesse devient:

$$
u=\frac{Q}{4 a} \frac{\operatorname{ch} \xi}{\operatorname{sh} \xi}+\frac{3 Q}{4 a}
$$

Le calcul qui fait intervenir un sh $\left(\pi R_{0} / 2 a\right)$ est facilité en développant en série ce terme, et en ne conservant que les deux premiers termes, compte tenu du fait que $R_{0} / a \ll 1$.

On aboutit alors à l'expression :

$$
H_{0}-3_{ \pm}^{\prime} R_{0}=(a / 2 \pi) \log \left(a / \pi R_{0}\right)
$$

On a bien entendu $H_{0} \gg R_{0}$, de sorte qu'on peut transformer cette expression en :

$$
H_{0}=(a / 2 \pi) \log \left(a / \pi R_{0}\right)
$$

(4) Dans tout ce qui suit le symbole Log représentera celui des logarithmes népériens. 
Cette formule définit $a$, relié au débit de fuite par $Q=K a$. En introduisant $a=Q / K$, on aboutit pour les fuites à la formule:

$$
\frac{H_{0}}{R_{0}}=\frac{Q}{2 \pi K R_{0}} \log \frac{Q}{\pi K R_{0}}
$$

Une fois connu le rapport $H_{0} / R_{0}$ (donnée du projet), cette formule - très simple malgré son caractère implicite - permet de calculer le débit de fuite.

Elle serait applicable telle que dans le cas d'une galerie creusée au rocher, sans revêtement.

$\mathrm{Au}$ point de vue physique, tout se passe comme si les fuites créaient une nappe autour de la galerie. La pression qui provoque les fuites n'est plus alors égale à $H_{0}$, mais à $H_{0}-b$.

\section{$2^{\circ}$ Perméabilité hétérogène : Cas d'une galerie revêtue et injectée}

Mais, en général, les galeries en charge sont revêtues dès que la charge dépasse une certaine valeur, car on considère souvent - nous verrons ce qu'il faut en penser compte tenu des considérations qui vont suivre - que le revêtement contribue à réduire les fuites. Ensuite, le terrain est, pour les mêmes raisons, injecté au voisinage de la galerie, de sorte que la perméabilité du milieu n'est plus égale à $K$, mais $K_{1}<K$, au moins dans une certaine zone.

Il n'y a pas de difficultés particulières à étendre le résultat précédent au cas d'une galerie creusée dans un terrain caractérisé par plusieurs perméabilités, sous réserve qu'elles soient homogènes dans un cylindre de rayon défini.

Nous allons admettre:

- que l'écoulement des fuites reste radial au voisinage de la galerie. C'est d'autant plus vrai que la pression intérieure est plus élevée;

- que les zones où la perméabilité a été réduite par rapport à sa valeur naturelle sont caractérisées par les données suivantes :

$K_{n} \quad$ représente le coefficient de perméabilité de la zone $n$,

$R_{n} \quad$ représente le rayon intérieur de la zone,

$R_{n+1}$ représente le rayon extérieur de la zone $n$.

La perte de charge entre $R_{n}$ et $R_{n+1}$ se détermine alors par la formule:

$$
\Delta H_{n}=\frac{Q}{2 \pi K_{n}} \int_{R_{n}}^{R_{n+1}} \frac{d u}{R}=\frac{Q}{2 \pi K_{n}} \log \frac{R_{n+1}}{R_{n}}
$$

La perte de charge totale des horizons successifs est alors égale à :

$$
\Delta H=\Sigma \Delta H_{n}=\Sigma \frac{Q}{2 \pi K_{n}} \log \frac{R_{n+1}}{R_{n}}
$$

En particulier, si on considère un revêtement de perméabilité $K_{0}$, et une zone injectée de perméabilité $K_{1}$, on aboutit à :

$$
\Delta H=\frac{Q}{2 \pi}\left[\frac{1}{K_{0}} \log \frac{R_{1}}{R_{0}}+\frac{1}{K_{1}} \log \frac{R_{2}}{R_{1}}\right]
$$

et il reste à la limite de la zone injectée et du terrain naturel une pression égale à $H_{0}-\Delta H$.

On peut alors appliquer les résultats précédents, correspondant au cas d'une canalisation en terrain brut, mais en introduisant la pression $H_{0}-\Delta H$, et le rayon $R_{2}$. On aurait alors en adaptant à ce cas la formule (1) ci-dessus :

$$
\begin{aligned}
H_{0}-\frac{Q}{2 \pi}\left[\frac{1}{K_{0}} \log \frac{R_{1}}{R_{0}}+\frac{1}{K_{1}} \log \frac{R_{2}}{R_{1}}\right] & = \\
= & \frac{Q}{2 \pi} \bar{K} \log \frac{Q}{2 \pi R_{2}}
\end{aligned}
$$

Soit, en passant à une expression sans dimension après avoir posé :

$\mu_{0}=K / K_{0}$ (perméabilité relative du terrain et des horizons $\mu_{1}=K / K_{1}$ autour de la galerie)

$$
\lambda_{0}=R_{1} / R_{0} \quad \lambda_{1}=R_{2} / R_{1}
$$

$$
\begin{array}{r}
\frac{H_{0}}{R_{0}}-\frac{Q}{2 \pi K R_{0}}\left[\left(\mu_{0}-1\right) \log \lambda_{0}+\left(\mu_{1}-1\right) \log \lambda_{1}\right] \\
=\frac{Q}{2 \pi K R_{0}} \log \frac{Q}{\pi K R_{0}}
\end{array}
$$

Si $\mu_{0}=\mu_{1}=1$, on retombe bien sur la formule initiale, relative aux pertes en terrain homogène. La pression relative $H_{0} / R_{0}$ est seulement réduite par le revêtement et l'injection dans la proposition de $H_{0} / R_{0}$ à la valeur donnée par le premier membre de la formule ci-dessus.

\section{$3^{\circ}$ Application numérique}

a) Galerie sans revêtement ou injection

$$
\text { Prenons } H_{0}=90 \mathrm{~m} \quad R_{0}=1,50 \mathrm{~m}
$$

La formule (1) s'écrit alors, en introduisant les valeurs numériques :

$$
60=u \log u, \quad \text { en posant } Q / \pi K R_{0}=u \text {. }
$$

On obtient alors $u=Q / \pi K R_{0}=34$.

En adoptant une valeur du coefficient de perméabilité égale à $10^{-6} \mathrm{~m} / \mathrm{s}$ (de l'ordre de 5 à 10 Lugeon), on aboutit à une valeur des fuites égale à :

$Q=0,161 / \mathrm{s}$ par mètre de galerie, ou :

$Q=1601 / \mathrm{s}$ par kilomètre de galerie.

On obtient par ailleurs :

$$
a=160 \mathrm{~m} \quad b=0,22 a=35 \mathrm{~m}
$$

b) Galerie revêtue, non injectée

Adoptons les données suivantes:

$R_{0}=1,50 \mathrm{~m} \quad \mu=10 \quad$ (revêtement 10 fois moins $R_{1}=1,80 \mathrm{~m} \quad$ perméable que le terrain)

La formule (2) s'écrit alors, en introduisant les valeurs numériques

$$
60-(u / 2) \cdot 1,64=(u / 2) \log u
$$

toujours en posant $Q / \pi K R_{0}=u$ 
On obtient alors $u=25$, soit $Q=0,121 / \mathrm{s}$ par mètre de galerie, et tout se passe comme si la charge relative $H_{0} / R_{0}=60$ avait été réduite, au contact đu terrain, à :

$$
60-12,5 \times 1,64=60-20,5=39,50 \mathrm{~m}
$$

Le revêtement a donc, grossièrement, encaissé le $1 / 3$ de la pression, tandis que le terrain encaissait les $2 / 3$ de celle-ci. C'est un résultat que nous commenterons par la suite.

\section{c) Galerie avec «manchon» injecté}

Nous supposerons que le revêtement et la zone injectée présentent la même perméabilité, 10 fois plus faible que celle du rocher, et que la zone injectée a une épaisseur égale à $2 R_{0}$. Donc :

$\mu=10$

$$
\lambda=3 \quad \log \lambda=1,10 .
$$$$
60-9,9 .(u / 2)=(u / 2) \log u
$$

On obtient alors: $u=10$, ce qui conduit à :

$$
Q=0,0471 / \mathrm{s} \text { par mètre de galerie }
$$

Cette fois, le débit de fuite est réduit dans des proportions élevées. La réduction de la pression au contact de la zone injectée avec le rocher naturel est alors importante.

\section{3 - Les efforts mécaniques. Leur influence sur la perméabilité}

Le résultat ci-dessus est purement hydraulique. Il définit comment la pression à l'intérieur de la galerie se répartit entre les diverses composantes du système d'étanchéité : revêtement, zone injectée, terrain naturel. Suivant une conception que nous avions développée dans l'étude $\mathrm{n}^{\text {" }} 1$, en 1960 , cette répartition des pressions est étroitement conditionnée par la répartition des perméabilités des divers horizons, du revêtement au terrain naturel.

Mais, en réalité, les perméabilités ne sont pas indépendantes des efforts, pour le béton notamment. Nous allons essayer d'apprécier, au moins en ordre de grandeur, dans quelles conditions la perméabilité du béton est influencée par les fissurations dont il peut être le siège.

Nous admettrons, dans tout ce qui va suivre, que le béton n'est pas armé, ou, en tout cas, que les armatures n'encaissent pas une part prépondérante de la pression intérieure. Pour que cette dernière hypothèse soit réalisée, il serait nécessaire que la densité des armatures soit particulièrement élevée. Or, justement, dans les galeries en charge, on essaie de faire au maximum appel au rocher pour encaisser la pression, sans prévoir des dispositifs particuliers. En outre, le béton est déjà bien difficile à faire en galerie. La mise en place des armatures elles-mêmes, les complications que leur présence apporterait à l'opération déjà délicate représentée par la mise en place du béton, rendrait cette opération sans doute très onéreuse, voire partiellement inefficace si la qualité du travail n'est pas suffisante. Mais peut-on contrôler les travaux en galerie au point où on serait vraiment sûr qu'il n'en soit pas ainsi ?

\section{A) DÉFORMATION DU TERRAIN ET DU BÉTON}

Le béton reçoit donc directement la part de la pression intérieure correspondant à la perte de charge qu'il inflige à l'écoulement poreux le traversant. S'il n'est pas armé, il ne peut que transférer cette charge sur le rocher: il ne résiste pratiquement pas à la traction.

Celui-ci se trouve alors chargé :

- en surface, par la pression que lui transmet le béton;

- en profondeur, par la pression de courant qui correspond à la part de pression intérieure que le revêtement n'encaisse pas.

Or, dans une étude antérieure (j), nous avions trouvé que la déformation d'un cylindre mis en pression dans un milieu poreux infini est la même, que cette pression soit appliquée en surface ou transmise par l'intermédiaire de l'écoulement poreux qu'elle provoque. Il ne s'agit là, d'ailleurs, que d'un corollaire du principe de Bhratz.

Nous pourrons alors calculer ces déformations en considérant que la pression est appliquée en surface, cas qui est traité par la théorie de l'élasticité.

La déformation radiale $u$ d'un cylindre de rayon $R_{0}$ creusé dans un milieu indéfini de coefficient d'élasticité $E$, chargé en surface par une pression $p$ est donnée pratiquement par l'expression $\left(^{6}\right)$ :

$$
u=p R_{0} / E=p D / 2 E
$$

L'épaisseur des fissures atteindra la valeur donnée par la déformation circonférentielle, soit $2 \pi u$.

\section{B) INFLUENCE DES FISSURES SUR LA PERMÉABLLITÉ DU BÉTON}

Les fuites à travers Ie béton résulteront alors :

- de sa microperméabilité, caractérisée par un certain coefficient de perméabilité, en général très faible;

- de sa macropcrméabilité, résultant elle-même:

- de l'existence de fissures «normales», reprises, nids de cailloux dans le matériau.

Ce sont ces deux phénomènes qui sont pris en compte dans la définition de la perméabilité d'un revêtement en béton. C'est en raison de l'existence des «anomalies» que constituent les fissures (en fait inévitables pour le béton de galerie), que nous avons pris, dans un des exemples numériques ci-dessus, un coefficient de perméabilité assez élevé, soit $10^{-7} \mathrm{~m} / \mathrm{s}$.

- de l'existence de fissures provoquées par la déformation du béton sous l'influence de la pression que reçoit le revêtement.

C'est ce dernier point que nous allons maintenant examiner.

\section{Calcul du débit de fuite en présence de fissures.}

Soit $L$ l'épaisseur du revêtement. Nous allons assimiler une fissure d'épaisseur $2 e$ à une fente de même largeur, sensiblement radiale, donc de longueur $L$.

(5) M. Bouvard, thèse de Doctorat (1967)

(6) En fait, la formule exacte contient, par rapport à celle-ci, un terme, correctif, voisin de 1, que nous négligerons. 
L'écoulement sera supposé laminaire. On sait qu'un écoulement est laminaire dans une conduite circulaire tant que le nombre de Reynolds est inférieur à des valeurs de l'ordre de 2400 . Or le nombre de Reynolds, pour de l'eau à $20^{\circ}$, est égal à, (en unités C.G.S.) :

$$
R=100 U D
$$

Si on admet que la valeur limite du nombre de Reynolds est la même pour des fissures, en remplaçant le diamètre du tuyau par l'épaisseur de celle-ci, on aurait pour l'écoulement laminaire limite dans une fissure de lordre du millimètre :

$$
10 U<2400
$$

On voit donc que la valeur limite de 2400 ne serait atteinte que pour des vitesses de l'ordre de $2 \mathrm{~m}$ et plus qui ne sont pas à considérer, compte tenu des résultats obtenus ci-après.

\section{a) Cas d'une seule fissure}

L'application des lois du frottement laminaire dans la fissure conduit alors à l'expression suivante :

$$
Q=(g / \nu) i \mathscr{2} / 3 e^{3}
$$

$Q=$ débit de la fissure, par unité de longueur;

$\nu=$ coefficient de viscosité cinématique du fluide (pour l'eau, $\nu=10^{-2} \mathrm{CGS}$ à $20^{\circ}$ );

$2 e=$ épaisseur de la fissure;

$i=$ perte de charge unitaire.

$\mathrm{Si}$ on appelle $\Delta H$ la perte de charge sur la longueur $L$ de la fissure, on obtient par conséquent, en prenant comme unité le $\mathrm{cm}^{3} / \mathrm{s}$ par $\mathrm{cm}$ de longueur de la fissure pour les débits, le centimètre pour $e$ :

$$
Q=10^{5}(\Delta H / L) \% e^{3}
$$

\section{b) Cas de plusieurs fissures totalisant} une certaine épaisseur.

L'épaisseur des fissures - supposées égales - est alors égale à $2 \mathrm{e} / \mathrm{n}$. Le débit total sera égal à $n$ fois le débit de chacune d'elle, ce qui donnera:

$$
Q=10^{5}(\Delta H / L) \text { 济 }\left(e^{3} / n^{2}\right)
$$

\section{$2^{\circ}$ Calcul de la perméabilité équivalente}

Nous allons comparer le débit ainsi obtenu à celui qui passe par percolation à travers un béton «normal », compte tenu de sa microperméabilité et des fissures «naturelles». Soit $k$ le coefficient de perméabilité de celui-ci. Ce débit est égal à :

$$
Q=S V=10^{4} \pi D k(\Delta H / L)
$$

en $\mathrm{cm}^{3} / \mathrm{s}$ par centimètre de longueur de revêtement.

Après fissuration, le débit total atteint donc:

$$
Q^{\prime}=10^{4} \cdot \pi D k \cdot(\Delta H / L)+10^{5}(\Delta H / L) \%\left(e^{3} / n^{2}\right)
$$

Définissons un nouveau coefficient de perméabilité $k^{\prime}$ par les fuites dues à la perméabilité naturelle et aux fissures de déformation. On aurait, par définition de $k^{\prime}$ :

$$
Q^{\prime}==10^{4} \cdot \pi D k^{\prime}(\Delta H / L)
$$

Soit :

$$
k^{\prime}=k+(e / \pi D)^{2 \%}(e / n)^{2}
$$

L'ordre de grandeur de $e$ est donné par les considérations que nous avons développées ci-dessus. L'épaisseur totale des fissures serait ainsi de l'ordre de $p \pi D / E, p$ représentant la pression dans la galerie, exprimée dans la même unité que $E$.

En reportant dans l'expression de $k^{\prime}$ ci-dessus, on obtient :

$$
k^{\prime}=k+\frac{20 \mathrm{p}}{6 E}\left[\frac{p}{2 E} \frac{\pi D}{n}\right]^{2}
$$

\section{Application numérique.}

Considérons le cas où :

$p=8 \mathrm{~kg} / \mathrm{cm}^{2} \quad, \quad E=30000 \mathrm{~kg} / \mathrm{cm}^{2} \quad, \quad D=3 \mathrm{~m}$

On obtient alors:

$$
k^{\prime}=k+1,4 \cdot 10^{-5} \cdot n^{-2}
$$

Il est bien difficile d'adopter une valeur de $n$ qui ne soit pas arbitraire. Néanmoins, nous conduirons le calcul avec $n=3$, ce qui pourrait correspondre à une fissure en calotte et deux fissures à la jonction piedroit galerie. On obtiendra dès lors :

$$
k^{\prime}=k+5 \cdot 10^{-6}
$$

On notera que, si la perméabilité naturelle $k$ du béton est de l'ordre de $10^{-i}$, elle se trouve considérablement augmentée par la fissuration: le second terme de l'expression ci-dessus est de l'ordre de 50 fois le premier.

\section{Remarques}

a) Il est bien certain que ce calcul ne peut que prétendre représenter un ordre de grandeur.

Le calcul lui-même de l'écoulement dans une fissure est correct. Mais, appliqué ici, il ne prend pas en compte d'incidence de l'écoulement, en aval, dans le rocher: le débit de fuite est concentré à l'endroit où la fissure s'arrête sur le rocher. La vitesse y est alors élevée, ce qui provoque, par rapport au calcul, l'apparition d'une perte de charge supplémentaire, jusqu'à ce que le débit soit uniformisé sur le pourtour du revêtement. Le phénomène est moins accentué si, localement, le revêtement est décollé du rocher (ce qui est sans doute partiellement le cas, au moins au voisinage de la fissure dans le béton).

$\mathrm{Si}$ la circulation dans le rocher s'opère surtout dans des fissures (ce qui constitue le cas certainement le plus fréquent), celles-ci s'ouvriront sous l'influence de la pression de l'écoulement. Il est alors raisonnable de penser qu'elles «fixeront» la position des fissures dans le béton, qui constitueront le simple prolongement de celles du rocher. L.e calcul ci-dessus cadrcrait alors micux avec la réalité.

b) Nous avons dit ci-dessus qu'il n'était pratiquement pas possible d'armer le revêtement d'une galerie pour qu'elle puisse résister à la pression intérieure totale. Mais une armature de répartition, beaucoup plus légère, contribuerait probablement à répartir la fissuration, donc à augmenter $n$. Or, le débit de fuite varie de façon inversement propor- 
tionnelle au carré de $n$, et il diminue donc rapidement si $n$ augmente. C'est sans doute l'avantage essentiel qu'on pourrait tirer de l'utilisation des armatures. Mais à quel prix et dans quelles conditions réelles?

c) Reste maintenant la question de la perméabilité relative du revêtement et du terrain. Comme nous l'avons indiqué dans l'étude $n^{\circ} 1$, c'est elle qui détermine l'effet réel du revêtement, à la fois sur les fuites et sur la part des pressions prise par le rocher ou le béton.

L'application numérique indiquée ci-dessus permet de constater que l'ordre de grandeur de la perméabilité du béton descend nécessairement à des valeurs de l'ordre de $10^{-6} \mathrm{~m} / \mathrm{s}$, et ceci avec un coefficient d'élasticité du rocher pas tellement pessimiste. Si celui-ci est plus déformable, le béton après fissuration sera encore plus perméable. Mais alors de deux choses l'une:

- ou bien le rocher a une perméabilité de l'ordre de $10^{-i} \mathrm{~m} / \mathrm{s},-$ beaucoup plus faible que celle du béton et le revêtement ne jouera qu'un rôle très modeste, soit dans la limitation des fuites, soit dans les efforts qu'il recevra: la perte de charge qu'il provoque est très réduite, et il se bornera alors à suivre la déformation du terrain;

- ou bien le rocher a une perméabilité de l'ordre de $10^{- \text {- }}$ lui aussi, ou supérieure; mais il sera alors indispensable de prendre des mesures pour diminuer ce coefficient de perméabilité : les fuites seraient par trop importantes; c'est uniquement dans le cas où le rocher serait peu déformable, mais perméable (deux propriétés relativement contradictoires) que le revêtement apporterait une contribution notable à l'équilibre de la galerie.

\section{4 - Hypothèses et réalités - Conclusions}

Naturellement, les calculs présentés ci-dessus n'ont pu être menés que moyennant toute une série d'hypothèses, sans lesquelles ils auraient été impossibles.

\section{A) LE CALCUL DES FUITES EN TERRAIN SEC. LES EFFETS DE L'INJECTION}

Le réseau qui définit les trajectoires des fuites est « exact 》 dans le cadre des hypothèses de base. Celles-ci consistent essentiellement à admettre que l'écoulement dans le rocher est assimilable à un écoulement microporeux, alors que l'eau circule avant tout par des fissures macroscopiques. Mais, à l'échelle de plusieurs dizaines de mètres, l'hypothèse d'une microperméabilité répartie, équivalente, est certainement loin d'être absurde. Aussi, on ne voit pas très bien par quoi on pourrait la remplacer.

L'assimilation de la section droite de la galerie à une équipotentielle de l'écoulement ne soulève aucune difficulté, et peut sans doute être considérée comme «rigoureuse»: la pression est constante sur une équipotentielle, et constante à la périphérie d'une galerie.

Les hypothèses introduites pour tenir compte de l'injection: réduction de la perméabilité avec un coefficient constant, dans un rayon cylindrique, représentent une manière de prendre en compte l'effet de l'injection qui, là encore, ne pourrait être remplacée par aucune autre hypothèse ayant une valeur générale, et plus vraisemblable.

Concrètement, la difficulté essentielle réside dans la mesure du coefficient de perméabilité du rocher. La dispersion des résultats est souvent élevée, et, comme nous l'avons montré dans une étude précédente (La Houille Blanche, $n^{\circ} 7 / 1969$ ) on ne sait même pas très exactement ce qu'on fait si la mesure s'effectue en terrain sec.

En tout cas, les hypothèses adoptées pour mener le calcul n'ajoutent pratiquement pas d'incertitudes par rapport à celles qu'on fait implicitement en définissant la perméabilité du rocher par un coefficient unique, et en admettant qu'on peut atteindre ce coefficient par les mesures telles qu'elles sont pratiquées couramment. Le degré de validité du résultat donné par la formule (2) est sans doute élevé, comparativement à ces difficultés.

\section{B) LE CALCUL de la PERTE D'ÉTANCHÉIté DU BÉTON SOUS L'EFFET DE LA PRESSION}

$\mathrm{Si}$ le calcul théorique de l'écoulement laminaire entre deux plans est rigoureux, les hypothèses introduites pour l'appliquer à l'écoulement dans les fissures du revêtement, puis la détermination de l'épaisseur globale des fissures pour le calcul des déformations du rocher à partir du théorème de Bhratz, sont beaucoup plus discutables. On n'a pas beaucoup de critères pour déterminer le nombre $n$ de fissures, qui joue un rôle non négligeable dans le calcul.

Un raisonnement très simpliste permet d'ajouter quelques arguments assez précis.

$\mathrm{Du}$ fait des caractéristiques du béton de galerie, il est certain qu'il se fissurera, si les fissures du rocher se dilatent, d'une façon sans doute très analogue. Compte tenu de sa résistance à la traction pratiquement nulle, le béton n'a pas de résistance autonome. $\mathrm{Si}$, conformément au résultat classique, le rocher est soumis sur une galerie circulaire à une compression de direction tangentielle (par report des efforts radiaux éliminés par la perforation), le béton, lui, n'est soumis à aucune précontrainte. On s'est rendu compte que les essais pratiqués vers les années 1950 , pour mettre en précontrainte le revêtement, ne donnaient pas le résultat escompté, et ils ont été abandonnés.

Que représente alors une épaisseur de béton - fissuré comme le rocher - de quelques décimètres, si l'eau doit parcourir plusieurs dizaines de mètres pour perdre sa pression? De toute façon, le raisonnement «boucle» sous un autre aspect: si le béton est étanche, il appuie sur le rocher qui se déforme alors comme une structure chargée en surface. Le calcul de la fissuration du béton, à partir de la formule $u=p R_{0} / E$ redevient alors valable, et avec lui le calcul de la «perméabilité équivalente » du revêtement. On se gardera seulement de donner au résultat numérique obtenu une valeur très précise.

\section{C) LE CARACTÈRE PLAN DE L'ÉCOULEMENT}

C'est bien sûr une hypothèse indispensable à la conduite du calcul. Mais, avons-nous dit, les conditions d'équilibre d'une galerie en charge - et les fuites qui en sont la conséquence - résultent d'un équilibre hydrogéologique complexe. 
Tout dépend alors des conditions aux limites «au large» pour l'écoulement des fuites dans le terrain.

- Si ces conditions sont telles que les fuites peuvent s'écouler sans difficultés une fois franchi le voisinage de la galerie, l'hypothèse de l'écoulement plan dans le terrain sera voisine de la réalité, et le calcul restera représentatif.

- Si, au contraire, l'eau qui a traversé la paroi du tunnel a des difficultés pour sortir du terrain, le cas sera bien différent.

Pour préciser cette distinction, nous introduirions la cote du plan de charge nécessaire à l'écoulement des fuites globales, après la galerie et compte tenu des conditions hydrogéologiques d'ensemble dans le massif. Si cette cote est nettement plus basse que la cote du plan de charge dans la galerie, on serait dans le premier cas, sinon on se retrouverait dans le second.

Une nouvelle conclusion se dégage: le débit perdu ne dépendrait plus alors, pratiquement, des dispositions prises au voisinage de la galerie, et il resterait à peu près le même que la galerie soit revêtue ou non, que le revêtement soit injecté ou non. Le calcul présenté ici perdrait alors de sa signification, mais dans un sens tel que les conclusions que nous en avons tirées sur la faible influence qu'exercent les précautions prises au voisinage de la galerie seraient encore renforcées.

Il est vrai qu'on reviendrait alors dans le cas de galeries en charge en présence d'une nappe: l'apparition de celle-ci résulterait encore des fuites, mais sa cote serait élevée même avec des débits faibles. Elle serait contrôlée par les propriétés du terrain loin en aval de la galerie.

\section{D) CONCLUSION}

Aussi quelques conclusions - importantes - se précisent ou se dégagent de toute façon à partir des calculs ou des considérations développées ci-dessus.

Ils confirment le caractère seulement relatif qu'il faut donner à la perméabilité du revêtement, et l'inffuence de ce paramètre sur les efforts reçus par le béton.

Ceux-ci, dans le sens galerie-rocher, ou dans le sens inverse, quand on vidange l'ouvrage, ou à la suite d'une baisse de pression consécutive à l'exploitation, sont étroitement fonction des perméabilités relatives. Ces efforts sont d'autant plus élev'és que le revêtement est plus étanche. La fissuration, que les contraintes de traction entrainent, devraient tendre à rapprocher la perméabilité du béton et celle du rocher.

En ce qui concerne les mesures à prendre pour définir les caractéristiques d'une galerie en charge:

$1^{\circ}$ Il est maintenant bien admis que les galeries en charge doivent être implantées le plus profondément possible, là où on peut compter sur la présence d'une nappe qui fait face à elle seule à la pression intérieure de la galerie.

Le revêtement ne joue plus de rôle au point de vue de l'étanchéité de la galerie.

C'est pour éviter les désordres, dus aux échanges d'eau entre la galerie et la montagne, que le revêtement est indispensable, mais essentiellement quand l'eau coule de la montagne vers la galerie: il pourrait se produire autrement un entraînement de matériaux par l'écoulement dans ce cas de fonctionnement, si le terrain est mauvais.

Linjection peut être utile pour consolider le revêtement, en lui permettant de constituer un bloc monolithe avec le rocher. Le revêtement encaissera d'autant mieux les efforts - permanents ou transitoires - même dans le sens rocher galerie, à la vidange par exemple.

On peut considérer que la solution originale appliquée à la galerie du Capivari, qui consiste à perforer systématiquement le revêtement pour l'équilibrer et limiter les efforts qu'il reçoit, présente de notables avantages si le terrain n'est pas mauvais au point que les éléments fins soient entrainés dans la galerie.

Bien entendu, cette opération devra être réalisée après exécution des injections, s'il faut en prévoir.

Accessoirement, on bénéficierait ainsi de débits supplémentaires si les débits normalement dérivés dans la galerie sont faibles, en drainant systématiquement le massif. Cette considération n'est pas toujours négligeable dans les pays arides: quelques centaines de litres par seconde, en étiage, peuvent être utiles vis-à-vis des débits de l'ordre du mètre cube seconde. Contrairement à ce que montrent les considérations à court terme, une venue d'eau en galerie représente souvent, à long terme, une excellente opération, même si les exécutants - et on les comprend - ne sont pas de cet avis. Poussant le raisonnement à l'extrême, on peut même dire que le rôle du revêtement est néfaste à ce point de vue: on perd une partie du débit (sans doute très faible) qu'on aurait par drainage de la nappe en galerie si les échanges d'eau entre terrain et galerie étaient libres.

$2^{\circ} \mathrm{Si}$ on ne peut éviter d'implanter l'ouvrage dans une zone où le terrain est sec.

La galerie se créera en quelque sorte sa propre nappe, au prix des fuites que nous avons calculées au cours des pages précédentes.

- Si la perméabilité du rocher est de l'ordre du Lugeon, on ne peut pratiquement rien faire pour réduire les fuites (qui sont alors d'un ordre de grandeur assez faible).

L'injection du terrain - si encore on pouvait l'exécuter ne réduirait pratiquement pas la perméabilité du terrain. Le ferraillage est trop onéreux. Le revêtement en béton se fissurera. Plus il sera imperméable, plus il recevra directement des efforts élevés (en réduisant les dimensions de l'auréole de fuites, caractérisée notamment par le paramètre que nous avons appelé $b$ ) et plus il sera obligé de solliciter l'appui du rocher pour les encaisser.

Seule l'injection de bourrage entre le revêtement et le rocher serait justifiée, mais plus pour des raisons mécaniques déjà évoquées ci-dessus.

- Si la perméabilité du rocher est beaucoup plus élevée (de l'ordre de 20 Lugeon par exemple), l'injection du rocher dans une certaine auréole autour de la galerie représente sans doute l'opération la plus avantageuse. Elle exerce un effet positif à beaucoup de points de vue. Elle permet de remplir les vides du terrain, ce qui constitue un bénéfice définitif sous plusieurs aspects : la perméabilité est réduite, la déformabilité aussi. A ce point de vue, on peut considérer que le revêtement lui-même représente seulement un ouvrage auxiliaire de l'opération d'injection : sans lui, elle serait impossible.

Si l'injection paraît donc constituer la seule solution envisageable, elle bute elle aussi sur des limites assez précises. D'abord, elle ne saurait réduire la perméabilité en dessous de celle correspondant à un Lugeon, de sorte que le débit de fuite donné par la formule(1), avec $K$ 
correspondant au Lugeon ( $\left.{ }^{\top}\right)$, représente une limite en dessous de laquelle il n'apparait pas possible de descendre par des moyens artificiels. Ensuite, si elle améliore les caractéristiques mécaniques du rocher, elle ne peut éviter sa fissuration sous l'influence de la pression dans la galerie ou de la pression dans l'écoulement poreux qui en est la conséquence. Cette fissuration, à son tour, va augmenter la perméabilité du terrain. Mais, évidemment, le remplissage des vides par les produits injectés reste définitivement acquis, et il ne peut que contribuer à améliorer les conditions générales de l'étanchéité, comme nous l'avons indiqué ci-dessus, mais dans une proportion très difficile à estimer.

Ces limites apparaissent plus rapidement, pour les très

(7) $K$ de l'ordre de 1 à $2 \cdot 10^{-7} \mathrm{~m} / \mathrm{s}$. Encore ce résultat ne pourrait être obtenu sur toute la longueur de la galerie que moyennant des soins particulièrement attentifs et des dépenses sans doute très élevées. hautes pressions en tout cas. Les essais, exécutés pour l'établissement du puits non blindé du Capivari, ont montré que l'injection ne servait pratiquement à rien si on mettait la galerie en charge à une pression de l'ordre de grandeur de la contrainte naturelle du rocher. Au-delà, le rocher «claque», les fissures s'ouvrent, et l'eau passe indépendamment des injections. Mais ce phénomène n'intervient que pour des pressions très élevées, très au-delà de celles utilisées couramment pour les galeries en charge «normales $\gg$.

De toutes façons, insistons une fois de plus sur le fait que les phénomènes dépendent des facteurs hydrogéologiques complexes. Seule leur étude - parfois difficile permet de savoir dans quel cas on se trouve, quelles théories ou formules doivent être utilisées pour préciser les dispositions à prendre. De toutes façons, le « nœud » du problème est déplacé bien au-delà du voisinage immédiat de la galerie.

\section{Abstract \\ Dry-ground leakage from power tunnels Respective effects of the tunnel lining, grouting operations and ground features}

Author's report is the latest in a series of research reports published in La Houille Blanche over a period of more than ten years in collaboration with other authors on the subject of unlined power tunnel equilibrium, among which notably on the theoretical and experimental research carried out on the unlined shaft at the Capivari power plant, which operates under a world record static pressure of 450 metres.

In the most frequent cases, and as a general rule whenever possible, power tunnels are installed at sufficient depth underground to ensure that the groundwater pressure exceeds the tunnel pressure so that, if anything, the tunnel receives water from its environment when in service.

Tunnels in a permeable medium not containing pressurized groundwater are a different case, however, as leakage is the only form of exchange that is liable to take place. This is the subject of Author's report, in which he attempts to calculate the possible leakage rates and establish how leakage can best be reduced

In the first part of the report, the theoretical characteristics of flow originating in the assumed homogeneous and isotropic rock medium as a result of tunnel pressure are determined and several leakage rate formulae derived, which allow for permeability of the surrounding medium and the presence or absence of tunnel lining.

Next, the forces causing leakage head loss are investigated, and it is shown that the resulting deformation of the tunnel lining will eventually crack it, thus considerably impairing its effectiveness in limiting losses (although this might be avoided by a very heavy lining, Author considers this to be a costly and somewhat doubtful solution).

The only thing left, therefore, is to rely on ground impermeability to provide an effective barrier. This can be improved by grouting, but the effectiveness of present methods is restricted to fairly definite limits associated with operational difficulties or deformation of the ground, even after grouting.

As a final conclusion, therefore, ideal impermeability of the considered type of structure would appear to be unattainable except if there were a strict ruling that power tunnels only be sited in areas known to contain a rock aquifer, i.e. beyond the stope of the present study. 